\title{
Treating Depression with ECT: An Objective Review
}

\author{
Basem Gohar ${ }^{1,2}$, Christa R. Winter ${ }^{1}$, Mark Benander ${ }^{3}$, Barbara Mandell ${ }^{1}$, \\ Cassandra Hobgood ${ }^{2}$, Keith Z. Brewster ${ }^{4}$ \\ ${ }^{1}$ Department of Psychology, Springfield College, Springfield, USA \\ ${ }^{2}$ Department of Psychiatry, Baystate Health, Springfield, USA \\ ${ }^{3}$ Department of Psychology, Baypath College, Longmeadow, USA \\ ${ }^{4}$ Department of Health and Exercise Science, University of British Columbia, Kelowna, Canada \\ Email: bgohar@springfieldcollege.edu
}

Received September 27 $7^{\text {th }}$, 2012; revised October 29 ${ }^{\text {th }}$, 2012; accepted November $8^{\text {th }}, 2012$

\begin{abstract}
The current study examined the efficacy of Electroconvulsive Therapy (ECT) as a treatment method for unipolar and bipolar depression using an objective measure through a retrospective chart review. First, this article discusses the history of ECT as well as issues in psychiatric diagnoses. Patients' progress in this study was measured by the hospital's psychiatrists as well as through the self-report measure, Clinically Useful Depression Scale (CUDOS). The sample consisted of 22 female and 8 male depressed inpatients and outpatients. A $2 \times 2$ mixed ANOVA revealed a significant interaction, showing improvement from pre-treatment to post-treatment in both genders. In post-treatment, female patients showed significantly more improvement than male patients. This study suggests that ECT results in depression reduction, especially in female patients. In addition, the CUDOS has shown to be a simple and effective self-report measure in assessing progress of depression including complex treatments, such as ECT. Recommendations for future ECT studies include: controlling for comorbidity and medication by obtaining a larger sample size to categorize patients according to medication type and dosage.
\end{abstract}

Keywords: Electroconvulsive Therapy; Depression; Self-Report Measure; Objectivity

\section{Introduction}

Depression is a mental disorder that is currently on the rise. Kessler, McGonagle, Nelson, Hughes, Swartz, and Blazer (1994) conveyed that approximately one in five individuals in the general population face at least one major depressive episode. Moreover, Kessler, Aguilar-Gaxiola, Alonso, Chatterji, Lee, and Üstun (2009) indicated in an analysis of the National Comorbidity Survey Replication that the prevalence of the disorder among adults in the United States is $16.2 \%$, which is the most common and costly illness. Additionally, Murray and Lopez (1996) indicated that major depressive disorder (MDD) was listed as the fourth ranked cause of premature death worldwide.

There are different types of depression as listed in The Diagnostic and Statistical Manual of Mental Disorders (DSM-IVTR; American Psychiatric Association, 2000). Some of the symptoms of depression include feeling sad or tearful, change in appetite, sleep and energy, inability to concentrate, suicidal ideations, feeling worthless, loss of interest or pleasure in activities which used to give pleasure, and psychomotor agitation. Causes of depression might differ for each individual. Some researchers suggested that depression is solely genetic-based (Campbell \& MacQueen, 2006; Hasler, Drevets, Manji, \& Charney, 2004; Penttila et al., 2004; Ressler \& Mayberg, 2007; Ruhé, Mason, \& Schene, 2007). Other researchers suggested that it is due to a joint contribution of genetic and environmental factors such as major life stressors (Cervilla et al., 2007; Duman \& Monteggia, 2006; Kendler, Gatz, Gardner, \& Pederse, 2006; Kim, Stewart, Kim, Yang, \& Shin, 2007; Rot, Mathew, \& Charney, 2009). Rot et al. concluded that depression is caused by a cumulative impact of genetics, adverse events in childhood and ongoing or recent stress. These findings portray that gene-environment interactions could better predict the risk of developing depression better than genes or environment alone.

Electroconvulsive therapy (ECT) is considered to be a last resort in treating depression; after failing to see results with medication and psychotherapy. Nevertheless, ECT is considered as one of the most effective treatments for depression (American Psychiatric Association, 2000; Sackeim, Prudic, Fuller, Keilp, Lavori, \& Olfson, 2006; Yatham et al., 2010). It has been stated, "The results of ECT in treating severe depression are among the most positive treatment effects of all of medicine” (Dukakis \& Tye, 2006: p. 132). Di Pauli and Conca (2009) described ECT as a required, induced suprathreshold stimulus in order to achieve therapeutic seizure. According to Beyer, Glenn, and Weiner (1998), ECT has proved to be effecttive since the 1930s. Keddy and Erdberg (2010) noted that ECT has been on the rise, with more than 100,000 Americans receiving treatment annually and approximately up to 20 times that many globally. ECT, however, is also considered to be one of the most controversial types of treatment in mental health (MacQueen, Parkin, Marriott, Begin, \& Hasey, 2007). Despite the treatment's efficacy, persistent fears, misunderstandings, and stereotypes continue.

\section{ECT Overview}

\section{History of ECT}

The notion behind inducing seizure as a treatment was exploited in 1934 (Beyer et al., 1998). Neuropsychiatrist, Ladislas Joseph von Meduna posited that individuals with epilepsy were saved from psychotic symptoms of schizophrenia (Fink, 1984). 
Meduna speculated that the use of convulsions in schizophrenic patients would reduce symptoms. The initial use of convulsion was done pharmacologically, using intramuscular injecttions of Camphor and Metrazol, which promoted seizures. Fink elucidated that Meduna's clinical trials demonstrated a significant decrease in psychotic symptoms. However, it was later revealed that there was no significant correlation between epilepsy and psychosis. Additionally, it was found that pharmacological methods to induce seizures had copious side effects.

Neuropsychiatrists, Ugo Cerletti and Lucio Binni introduced the use of electricity to induce seizures in 1937 (Accornero, 1988). They discovered that using electricity was more effective and safer than pharmacological agents. Babigian and Guttmacher (1984) acknowledged the popularity of ECT treatment, however the reputation of the treatment began to diminish during the 1950s. They credited the insufficiency of the treatment to the discoveries of medications treating depressive, manic, and psychotic symptoms. In addition to discoveries of medication, it was believed that the media was a leading cause for the disrepute of the treatment. Films, such as One Flew Over the Cuckoo's Nest, which was produced in 1975, showcased ECT as an act of ruthless behavioral punishment (Jenkusky, 1992).

\section{ECT Today}

Presently, ECT is slowly earning a positive reputation and is used for a variety of mental health reasons (Beyer et al., 1998). ECT is used mainly for patients suffering from unipolar and bipolar depression, psychotic disorders, as well as with pregnant patients with severe symptomology in the event that the necessary medication is contraindicated for fetal health. While ECT has shown to be successful, there are a variety of factors that must be accounted for to avoid adverse effects (Le Strat \& Gorwood, 2007). Beyer et al. (1998) reported that the mortality rate in ECT is low. The researchers also reported that no contraindications have been researched for ECT. However, there are factors that could increase the risk of complications and adverse effects medically and cognitively.

From a medical perspective, ECT could be considered higher in risk if there are any irregularities found in the brain, such as detecting a tumor or hematoma (Beyer et al., 1998). Other conditions include recent myocardial infractions, intracerebral hemorrhage, unstable vascular aneurysm or malformation, or anesthetic complications. The researchers highlighted that a significant positive correlation is found between mortality rate and those with comorbid medical diseases, specifically cardiovascular and respiratory diseases.

Cognitively, the researchers outlined three potential impairments (Beyer et al., 1998). First is postictal confusion, which is a brief state of confusion that lasts from minutes to hours after patients awaken from ECT treatment. Daniel and Crovitz (1986) identified a variety of factors that could affect postictal confusion. Risks of postictal confusion are higher when sine waves, stimulus intensity and frequency in treatment increase. Advancing age was also seen as a predictor. However, a metaanalysis by Flint and Gagnon (2002) found a positive correlation between ECT efficacy and advancing age. They explained that age is not the cause of postictal confusion; however, it is the age-related neurological conditions (e.g. dementia) that are attributed to the cognitive deficits. Thus, proper evaluations are necessary for older patients prior to treatment.

The second type of cognitive impairments that could occur with ECT is interictal confusion, which Beyer et al. (1998) described as a more serious state of confusion that develops into a state of delirium. Interictal confusion however is found to be uncommon. Memory dysfunctions, such as retrograde amnesia are also possible outcomes. In addition, electrode placements in ECT may contribute to short-term or long-term adverse effects. For example, unilateral placement poses less postictal deficits post treatment, however, is normally found to be less effective than bitemporal (Sackeim et al., 2006). While bitemporal has been shown to be more effective, cognitive outcomes could be more potent and long lasting, such as retrograde amnesia.

\section{Diagnosis}

Vis-à-vis diagnosis and progress examinations in a psychiatric capacity, psychiatrists are experts and skilled professionals who are meticulous in their assessments. They gather the necessary information directly through interviews with patients and indirectly through case discussions in multidisciplinary treatment teams as well as reviewing prior documents pertinent to the patient. Consequently, this has raised concerns for potential subjectivity, as detecting and perceiving symptoms of mental disorders can be exceptionally imprecise. Engel (1977), the founder of the biopsychosocial model for medicine explained that the field of psychiatry is ambiguous and "in contrast, the rest of medicine appears neat and tidy. It has a firm base in biological sciences and its command, and a record of astonishing achieving in elucidating mechanisms of disease and devising new treatments” (Engel, 1977: p. 129).

The concern of subjectivity remains an issue in more recent literature. Bloch, Ratzoni, Mendlovic, Gal, and Levkovitz (2005) criticized the inconsistencies in determining maximal medical improvement (i.e. returning to baseline through treatment) of patients with mental disorders across the literature. Therefore, measuring progress, especially with a treatment such as ECT would benefit from the inclusion of an objective and standardized approach in addition to the psychiatrists' assessments. Zimmerman and Mattia (2001) accentuated the importance of accurate, reliable, valid, and accessible measures in evaluating the quality and efficiency of care in clinical practice. Thus, standardized measures such as self-reported instruments that measure fluctuating affective states in psychiatric patients are necessary. The researchers described self-report questionnaires as a cost-effective option because they are inexpensive and require less time for completion. In addition, the researchers pointed out that the results of self-report questionnaires correlate highly with the ratings of clinicians.

Among the newer self-report questionnaires used today is the Clinically Useful Outcome Depression Scale (CUDOS, Zimmerman, Chelminski, McGlinchey, \& Posternak, 2008). The questionnaire consists of 18 items. Of those items, 16 assess all of the DSM-IV-TR (American Psychiatric Association, 2000) inclusion criteria for MDD and dysthymic disorder. The two remaining items examine psychosocial impairment and quality of life. It takes approximately three min to complete by the patient and about $15 \mathrm{~s}$ to score by the clinician, which works well in the ECT treatment setting, where it would not aggravate the patients prior to the procedure. Psychometric properties of the CUDOS are discussed in the methods section.

The present study was designed to objectively examine symptoms of depression in previous patients who were under- 
going ECT. Initial and final scores of the CUDOS in a course of treatment were gathered retrospectively from depressed patients who have undergone ECT.

\section{Methods}

The Institutional Review Boards of Baystate Health (BH) as well as Springfield College in Springfield, MA approved this study.

\section{Sample}

Data were collected from archived records of patients at $\mathrm{BH}$ from September 2010 to January 2012. The sample consisted of 30 former inpatients and outpatients at $\mathrm{BH}$, who required ECT for treatment due to depression, failed to reach maximal medical improvement by medication and therapy, and who received a minimum of four treatments in one series of ECT. In this study, patients were diagnosed with unipolar or bipolar depression.

\section{ECT}

Unilateral or bilateral (bitemporal) electrodes were placed on patients based on the psychiatrists' impressions prior to treatment using the MECTA 5000Q device at BH. Typically, patients received three treatments per week, as is the clinical custom at $\mathrm{BH}$ and in the United States.

\section{Measure}

In this study, the researchers examined previous scores of the CUDOS (Zimmerman et al., 2008) in former depressed patients of the hospital who had undergone ECT treatment. Zimmerman et al. tested the reliability and validity of the CUDOS on 1475 participants. Of those participants, $42.4 \%$ were diagnosed with MDD. The CUDOS displayed strong internal consistency in sample with alpha of .90 . The test-retest reliability of the CUDOS was established with 176 participants at baseline $(r=.92)$ and 33 participants during follow-up $(r=.95)$. Pertaining to validity, 200 participants were given a variety of mood disorder questionnaires including CUDOS to be completed. The researchers discovered that CUDOS was highly correlated with the Beck's Depression Inventory (Beck, Ward, Mendelson, Mock, \& Erbaugh, 1961) than with measures of other symptom domains, which indicates strong discriminant validity. The psychiatrists at $\mathrm{BH}$ favor the CUDOS and use the questionnaire as a part of the progress evaluations as it correlates with their evaluations and takes little time to complete by the patient and to score by the psychiatrists.

\section{Procedure}

The researchers created a raw score sheet. This sheet did not contain any Health Insurance Portability and Accountability Act (HIPPA) information pertaining to the patient, as it was de-identified information. The researchers organized the information obtained from the database of the hospital, which included: (a) codes given to patients (e.g. ECT1), (b) age, (c) gender, (d) pre-scores of CUDOS (Zimmerman et al., 2008), and (e) post-scores of the CUDOS. Pre-scores were the patients' outcome measures prior to their first ECT session in a course of treatment. Post-scores were their outcome measures prior to their last ECT session in a course of treatment.

\section{Statistical Analyses}

Descriptive statistics were used to describe participants in the study. A $2 \times 2$ mixed factorial Analysis of Variance (ANOVA) was computed to compare pretest and posttest scores of the CUDOS (Zimmerman et al., 2008) as well as gender. TukeyKramer post hoc tests were used to follow up significant findings

\section{Results}

Basic assumptions for conducting the mixed factorial ANOVA were met, except homogeneity of variance was not supported for the CUDOS (Zimmerman et al., 2008) scores post intervention $(p=.03)$. A total of 30 patients were selected from the archived records of the hospital; 22 (age $\mathrm{M}=51.78$; $\mathrm{SD}=$ 12.82) were female patients and 8 (age $M=53.38$; $S D=14.99$ ) were male patients. Descriptive statistics for age and number of treatments based on gender of the sample are presented in Table 1. Pre-scores and post-scores of the CUDOS and gender were analyzed using a $2 \times 2$ mixed factorial ANOVA. The independent group variable was gender (male and female). The repeated measures factor was test occasion (pretest and posttest). The dependent variable was depression, which was measured by the CUDOS. Mean scores of the CUDOS (pre and post) by gender are presented in Table 2 .

The $2 \times 2$ mixed factorial ANOVA revealed a significant interaction between test occasion and gender $\mathrm{F}(1,28)=5.87, p$ $=.02$. A summary of the $2 \times 2$ mixed factorial ANOVA is presented in Table 3. Due to the significant interaction, TukeyKramer post hoc tests were conducted. Three post hoc comparisons were significant. First, there was a significant improvement $(p<.05)$ from pretest $(\mathrm{M}=46.25$; $\mathrm{SD}=8.58)$ to posttest scores $(M=33.63$; $S D=20.23)$ in male patients. Similar findings were found in female patients, who also showed significant improvement $(p<.05)$ from pretest $(\mathrm{M}=49.68$; $\mathrm{SD}$ $=11.03)$ to posttest $(\mathrm{M}=23.32 ; \mathrm{SD}=12.44)$. Lastly, a significant difference $(p<.05)$ was found in posttesting, with female patients $(\mathrm{M}=23.32$; $\mathrm{SD}=12.44)$ showing significantly lower scores than male patients $(M=33.63$; $S D=20.23)$. No significant difference $(p>.05)$ was found in the pretest scores between male and female patients.

\section{Discussion}

The current study was designed to determine the effects of ECT on patients suffering from depression through a retrospecttive chart review in an objective approach by examining scores of the CUDOS (Zimmerman et al., 2008) prior to first and at the end treatment in a series. The results of the study showed a reduction in levels of depression, as indicated by the change of scores of the CUDOS after the completed ECT treatment. Thus, the results of this study support the efficacy of ECT in patients suffering from depression. Using a standardized method for each patient was helpful in this study when examining progress. Gilbody, House, and Sheldon (2002) reported that the use of standardized and quantifiable outcome measures is useful. However, they noted that they are rarely used in routine clinical practice.

It is important to note that the initiative behind using an objective measure is not to replace the psychiatrists' evaluations, 
Table 1.

Descriptive statistics of patients' age, number of treatments, and mean seizure length based on gender of the sample $(\mathrm{N}=30)$.

\begin{tabular}{ccccc}
\hline Variable & Mean & SD & Minimum & Maximum \\
\hline Age & & & 31 & 73 \\
Male & 53.38 & 14.99 & 33 & 77 \\
Female & 51.78 & 12.82 & 5 & \\
$\begin{array}{c}\text { Number of } \\
\text { Treatments }\end{array}$ & & & 5 & 11 \\
Male & 7.77 & 1.83 & 5 & 18 \\
Female & 7.75 & 3.79 & 4 & \\
\hline
\end{tabular}

Note. Male $\mathrm{n}=8$, Female $\mathrm{n}=22$

Table 2.

Descriptive statistics of patients' CUDOS Scores Pre and post ECT treatment in series $(\mathrm{N}=30)$.

\begin{tabular}{cccccc}
\hline Gender & $\mathrm{n}$ & Mean & SD & Minimum & Maximum \\
\hline Pre CUDOS & & & & & \\
Male & 8 & 46.25 & 8.58 & 32 & 65 \\
Female & 22 & 49.68 & 11.03 & 27 & \\
Total & 30 & 48.77 & 10.40 & & \\
Post CUDOS & & & & & 60 \\
Male & 8 & 33.63 & 20.23 & 3 & 44 \\
Female & 22 & 23.32 & 12.44 & 4 & \\
Total & 30 & 26.07 & 15.24 & & \\
\hline
\end{tabular}

Table 3.

$2 \times 2$ mixed factorial ANOVA comparing gender and test occasion of CUDOS before and after ECT treatment $(\mathrm{N}=30)$.

\begin{tabular}{cccccc}
\hline Source & SS & df & MS & F & p \\
\hline $\begin{array}{c}\text { Between } \\
\text { Groups }\end{array}$ & & & & & \\
Group & 69.23 & 1 & 6.23 & .59 & .45 \\
Error & 3273.22 & 28 & 116.87 & & \\
Within Subjects & & & & & \\
Occasion & 8918.00 & 1 & 8918.00 & 47.80 & .00 \\
A $\times$ B & 1107.33 & 1 & 1107.33 & 5.87 & .02 \\
Error & 5280.97 & 28 & 188.61 & & \\
\hline
\end{tabular}

but to simply reduce any form of subjectivity that may arise. Further, the process of ECT cannot be entirely left for standardized measures. For example, the psychiatrists determine the placement of the electrodes for each patient prior to treatment, a clinical judgement that is based on knowledge and experience. Due to the complexity of each case, the psychiatrists must select the best option for each patient; striving to deliver the lowest level of risk in conjunction with the highest degree of bene- fit. Other factors that psychiatrists must consider for ECT include, but are not limited to: electrode placement, age, stimulus intensity, sine wave intensity, treatment frequency, medication use, and medical conditions (Beyer et al., 1998).

The inclusion of standardized measures in the process of ECT however generates a number of benefits. A self-report measure as simple as The CUDOS (Zimmerman et al., 2008) provides a clear and visible objective measure to the patients and their loved ones in order to monitor progress. A standardized measure is also helpful in supporting the decision of the psychiatrists' progress assessments in two ways. It could support the decision to terminate treatment based on a patient reaching maximal medical improvement, which would be noticeable by viewing a reduction in the CUDOS scores. It could also support the decision to terminate treatment if a patient is not showing improvement, as the CUDOS scores would marginally decrease, remain the same, or increase.

The results of this study suggest that both male and female patients had significant improvement after ECT series. However, female patients showed more improvement than male patients, evidenced by the scores on the CUDOS (Zimmerman et al., 2008). This finding resembles the findings of Bloch et al. (2005), who determined that female schizophrenic patients had significantly more improvement than male schizophrenic patients after ECT treatments. Therefore, female patients with schizophrenia and depression show more improvement than male patients after ECT. Although depression diagnoses are classified as mood disorders and schizophrenia is classified as a psychotic disorder, the disorders share similarities in improvement using the same treatment. For example, Mahmoud et al. (2007) found improvement in both types of disorders using the same medication. They determined that the use of antipsychotic medications such as Risperdal has been effective in treating MDD.

Difference in improvement between genders however could be attributed to the difference in depressive symptoms presented based on gender. For example, Gorman (2006) examined the difference of displaying sadness between men and women. The researchers found that women displayed more visible emotions of sadness (e.g. crying) than men, who were more rigid. In another study, Winkler, Pjerk, and Kasper (2006) reported that men displayed depression in a more aggressive and irritable behavior than women. Thus, ECT might be more effective in treating symptoms displayed by women more than those displayed by men. Also, female patients might have shown better improvement based on the reports of the depressive symptoms. Winkler et al. reported that female patients had more visits to their doctor regarding symptoms of depression and anxiety than male patients. Therefore, female patients showing more improvement than male patients could be attributed to earlier intervention, more frequent consultations, and greater engagement with health professionals. As a result, the current finding questions the differences in manifestations as well as the chronicity of the depression between genders prior to seeking professional help. The possibilities listed above are speculations due to the lack of literature with regards to gender differences in ECT patients, specifically in patients suffering from depresssion. As a result, gender differences would benefit more exploration in future studies with more and equal numbers of male and female patients.

There were some limitations to this study. First, depressed patients might have presented different symptoms. Second, the 
duration of the disorder might have varied from one patient to another. Third, as this was a retrospective chart review, the researchers were unable to account for cognitive impairments that might have occurred during the treatments. Therefore, it is unknown whether cognitive deficits led to discontinuation of the full series prior to reaching maximal medical improvement for some patients. Despite the observed results of each patient, even if CUDOS (Zimmerman et al., 2008) scores displayed improvement, it is unknown if the patient did truly meet maximal medical improvement. Therefore, this study cannot conclude that patients overall reached maximal medical improvement. Alternatively, it can be concluded that patients, overall, showed improvement after ECT treatments.

Fourth, the selection process delimited the study by selecting patients who were diagnosed with a type of depression and received a minimum of four ECT treatments in a series. However, this study did not control for comorbid psychiatric disorders. Therefore, it is unknown if differences in progress were due to comorbidity. This could be the case as Afifi (2005) noted that patients with MDD commonly have a comorbid anxiety disorder. Lastly, this study did not control for medication during ECT treatment. Due to the nature of the treatment, it was difficult to control for medication as each patient had different circumstances medically and psychologically. Therefore, recommendations for future ECT studies include: controlling for comorbidity and medication, obtaining a larger sample size to categorize patients according to medication type and dosage.

\section{Conclusion}

In conclusion, the findings of this study support the efficacy of ECT in treating depression. More improvement was shown in female patients than male patients. Lastly, the CUDOS has shown to be a simple and effective self-report measure in assessing progress of depression including complex treatments, such as ECT. The measure is also helpful in providing a clear and objective approach to measure treatment outcome.

\section{REFERENCES}

Accornero, F. (1988). An eyewitness account of the discovery of electroshock. Convulsive Therapy, 4, 40-49.

Afifi, M. (2005). Gender differences in mental health. Singapore Medical Journal, 48, 385.

American Psychiatric Association (2000). Diagnostic and statistical manual of mental disorders (4th ed., text revision). Washington, DC: Author.

Babigian, H. M., \& Guttmacher, L. B. (1984). Epidemiologic considerations in electroconvulsive therapy. Archive of General Psychiatry, 41, 246-253. doi:10.1001/archpsyc.1984.01790140036005

Beck, A. T., Ward, C. H., Mendelson, M., Mock, J., \& Erbaugh, J. (1961). An inventory for measuring depression. Archive of General Psychiatry, 4, 561-571. doi:10.1001/archpsyc.1961.01710120031004

Beyer, J. L., Glenn, M. D., \& Weiner, R. D. (1998). Electroconvulsive therapy: A programmed text (2nd ed.). Arlington, VA: American Psychiatric Press.

Bloch, Y., Ratzoni, G., Mendlovic, S., Gal, G., \& Levkovitz, Y. (2005). Gender differences in electroconvulsive therapy: A retrospective chart review. Journal of Affective Disorders, 84, 99-102. doi:10.1016/j.jad.2004.10.002

Campbell, S., \& MacQueen, G. (2006). An update on regional brain volume differences associated with mood disorders. Current Opinion in Psychiatry, 19, 25-33. doi:10.1097/01.yco.0000194371.47685.f2

Cervilla, J. A., Molina, E., Rivera, M., Torres-González, F., Bellón, J. A., Moreno, \& Gutiérrez, B. (2007). The risk for depression con- ferred by stressful life events is modified by variation at the serotonin transporter 5HTTLPR genotype: Evidence from the Spanish PREDICT-Gene cohort. Molecular Psychiatry, 12, 748-755. doi:10.1038/sj.mp.4001981

Daniel, W. F., \& Crovitz, H. F. (1986). ECT-induced alteration of psychogenic amnesia. Acta Psychiatrica Scandinavica, 74, 302-303. doi:10.1111/j.1600-0447.1986.tb06247.x

Di Pauli, J., \& Conca, A. (2009). Impact of seizure duration in maintenance electroconvulsive therapy. Psychiatry and Clinical Neurosciences, 63, 769-771. doi:10.1111/j.1440-1819.2009.02028.x

Duman, R. S., \& Monteggia, L. M. (2006). A neurotrophic model for stress-related mood disorders. Biological Psychiatry, 59, 1116-1127.

Engel, G. L. (1977). The need for a new medical model: A challenge for biomedicine. Science, 196, 129-136. doi:10.1126/science.847460

Fink, M. (1984). Meduna and the origins of convulsive therapy. American Journal of Psychiatry, 141, 1034-1041.

Flint, A. J., \& Gagnon, N. (2002). Effective use of electroconvulsive therapy in late-life depression. Canadian Journal of Psychiatry, 47, 734-741.

Gilbody, S., House, A., \& Sheldon, T. (2002). Psychiatrists in the UK do not use outcomes measures. British Journal of Psychiatry, 180, 101-103.

Gorman, J. M. (2006). Gender differences in depression and response to psychotropic medication. Gender Medicine, 3, 93-109. doi:10.1016/S1550-8579(06)80199-3

Jenkusky, S. M. (1992). Public perception of electroconvulsive therapy: A historical review. Jefferson Journal of Psychiatry, 10, 2-11.

Keddy, P., \& Erdberg, P. (2010). Changes in the Rorschach and MMPI-2 after electroconvulsive therapy (ECT): A collaborative assessment case study. Journal of Personality Assessment, 92, 279-295. doi:10.1080/00223891.2010.481982

Kendler, K. S., Gatz, M., Gardner, C. O., \& Pederse, N. L. (2006). Personality and major depression. Archives of General Psychiatry, 63, 1113-1120. doi:10.1001/archpsyc.63.10.1113

Kessler, R. C., McGonagle, K. A., Nelson, C. B., Hughes, M., Swartz, M., \& Blazer, D. G. (1994). Sex and depression in the National Comorbidity Survey II: Cohort effects. Journal of Affective Disorders, 30, 15-26. doi:10.1016/0165-0327(94)90147-3

Kim, J. M., Stewart, R., Kim, S. W., Yang, S. J., \& Shin, I. S. (2007). Interactions between life stressors and susceptibility genes (5-HTTLPR and BDNF) on depression in Korean elders. Biological Psychiatry, 62, 423-428. doi:10.1016/j.biopsych.2006.11.020

Le Strat, Y., \& Gorwood, P. (2007). Mortality in electroconvulsive therapy. British Journal of Psychiatry, 191, 362. doi:10.1192/bjp.191.4.362

MacQueen, G., Parkin, C., Marriott, M., Bégin, H., \& Hasey, G. (2007). The long-term impact of treatment with electroconvulsive therapy on discrete memory systems in patients with bipolar disorder. Journal of Psychiatry and Neuroscience, 32, 241-249.

Mahmoud, R. A., Pandina, G. J., Turkoz, I., Kozik-Gonzalez, C., Canuso, C. M., Kujawa, M. J., \& Gharabawi-Garbaldi, G. (2007). Risperidone for treatment-refractory major depressive disorder: A randomized trial. Annals of Internal Medicine, 147, 593-603.

Murray, C., \& Lopez, A. (1996). The global burden of disease. Cambridge, MA: Harvard University Press.

Rot, M., Mathew, S. J., \& Charney, D. S. (2009). Neurobiological mechanisms in major depressive disorder. Canadian Medical Association Journal, 180, 305-313. doi:10.1503/cmaj.080697

Ruhé, H. G., Mason, N. S., \& Schene, A. H. (2007). Mood is indirectly related to serotonin, norepinephrine and dopamine levels in humans: A meta-analysis of monoamine depletion studies. Molecular Psychiatry, 12, 331-359. doi:10.1038/sj.mp.4001949

Sackeim, H. A., Prudic, J., Fuller, R., Keilp, J., Lavori, P. W., \& Olfson, M. (2006). The cognitive effects of electroconvulsive therapy in community settings. Neuropsychopharmacology, 32, 244-254. doi:10.1038/sj.npp.1301180

Winkler, D., Pjerk, E., \& Kasper, S. (2006). Gender-specific symptoms of depression and anger attacks. Journal of Men's Health and Gender, 30, 19-24. doi:10.1016/j.jmhg.2005.05.004

Yatham, L. N., Liddle, P. F., Lam, R. W., Zis, A. P., Stoessl, A. J., 


\section{B. GOHAR ET AL.}

Sossi, V., \& Ruth, T. J. (2010). Effect of electroconvulsive therapy on brain $5-\mathrm{HT}_{2}$ receptors in major depression. British Journal of Psychiatry, 197, 499-500.

Zimmerman, M., \& Mattia, J. I. (2001). A self-report scale to help make psychiatric diagnoses: The Psychiatric Diagnostic Screening
Questionnaire (PDSQ). Archives of General Psychiatry, 58, 787-94. Zimmerman, M., Chelminski, I., McGlinchey, J. B., \& Posternak, M. A. (2008). A clinically useful depression outcome scale. Comprehensive Psychiatry, 49, 131-140. doi:10.1016/j.comppsych.2007.10.006 